\title{
Frictional cooling of positively charged particles
}

\author{
Daniel Greenwald* and Allen Caldwell \\ Max Planck Institute for Physics, Munich, Germany \\ (Received 17 November 2011; published 29 February 2012)
}

\begin{abstract}
One of the focuses of research and development towards the construction of a muon collider is muon beam preparation. Simulation of frictional cooling shows that it can achieve the desired emittance reduction to produce high-luminosity muon beams. We show that for positively charged particles, charge-exchange interactions necessitate significant changes to schemes previously developed for negatively charged particles. We also demonstrate that foil-based schemes are not viable for positive particles.
\end{abstract}

DOI: 10.1103/PhysRevSTAB.15.024003

PACS numbers: 14.60.Ef, 29.27.- a, 41.75.Ak

\section{INTRODUCTION}

One of the focuses of research and development towards the construction of a muon collider is muon beam creation and preparation. The short lifetime of the muon necessitates preparation of a muon beam on time scales shorter than a microsecond. To achieve luminosities on the order of $10^{34} \mathrm{~cm}^{-2} \mathrm{~s}^{-1}$, as in the schemes of [1-3], requires a reduction of beam emittance-known as beam coolingby 6 orders of magnitude.

Simulations of frictional cooling [4] show that it can achieve the desired emittance reduction and potentially produce high-luminosity muon beams [3]. While other schemes have been investigated for production of a muon beam (most prominently ionization cooling [5-8] for a neutrino factory $[1,9]$ ), simulations have yet to demonstrate the potential to produce the beam qualities required of a muon collider. Furthermore, in simulation, frictional cooling has been shown to produce the desired luminosity for a muon collider with a bunch population smaller than considered in the above-cited collider studies. A reduced beam population is advantageous in reducing the background flux in the interaction region of the collider and therefore beneficially impacts detector design [10].

Previous work on frictional cooling, including both simulation and experiment [11], focused on cooling $\mu^{-}$ beams and assumed the cooling mechanism would be identical for $\mu^{+}$beams. However, positively charged particles, unlike negatively charged ones, participate in charge-exchange processes, and this greatly alters their cooling. In this paper, we present calculations and simulations of the effects of charge-exchange processes on the frictional cooling of positive particles. We also show that

\footnotetext{
*Corresponding author. deg@mpp.mpg.de

Published by the American Physical Society under the terms of the Creative Commons Attribution 3.0 License. Further distribution of this work must maintain attribution to the author(s) and the published article's title, journal citation, and DOI.
}

previous simulations of foil-based schemes are invalid for positive particles, and rule out the viability of such schemes.

\section{FRICTIONAL COOLING}

Frictional cooling reduces the energy spread and divergence of a beam by balancing retarding forces from interactions with a medium, with accelerating forces from an electric field to bring the beam to an equilibrium energy. Figure 1 shows the stopping power $S=(1 / \rho) d T / d s$, where $d T / d s$ is the energy loss per unit path length, and $\rho$ is the medium density, of helium for $\mu^{+}$and the acceleration power $(E / \rho)$ of an electric field of fixed strength $E$ on a particle of constant unit charge. The stopping power is velocity scaled ${ }^{1}$ [12] for $\mu^{+}$from the proton data given in [13]. The stopping power for $\mu^{-}$has a similar shape, though it is smaller in magnitude at energies below approximately $100 \mathrm{keV}$ [14,15]. When the accelerating power is larger than the stopping power, the particle is accelerated. When the reverse is true, the particle is decelerated. At the kinetic energies where the two powers are equal, particles are at an energy equilibrium. If the stopping power is greater than the accelerating power at energies above this equilibrium point and vice versa below it, then the point is stable and attractive.

For a particle of constant charge, two equilibrium points are created: a stable one ( $T_{\text {eq }}$ in Fig. 1) at an energy below that at which the stopping power peaks, and an unstable one at an energy above $\left(T_{\text {eq }}^{\prime}\right)$. Particles with $T<T_{\text {eq }}^{\prime}$ will be brought to $T_{\text {eq }}$, defining the frictional-cooling energy region.

Straggling of energy losses to the medium prevent a beam from becoming truly monoenergetic in a frictionalcooling scheme, inducing a spread of the beam energy around $T_{\text {eq }}$. A study of the frictional cooling of $\mu^{-}$in [4] found that the spread of the energy distribution of a cooled

\footnotetext{
${ }^{1}$ Except where noted, data for $\mu^{+}$interactions in this paper are velocity scaled from proton data.
} 


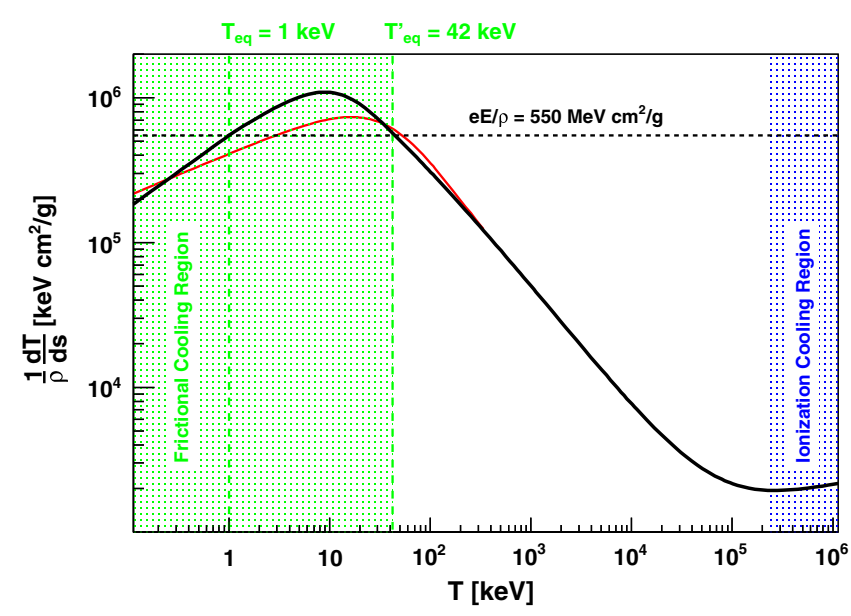

FIG. 1. Stopping power of helium for $\mu^{+}$(black, velocity scaled from [13]) and $\mu^{-}$(red, velocity scaled from [14]) where it deviates from $\mu^{+}$, and the acceleration power of an electric field of fixed strength $E$ for a particle of constant unit charge as functions of particle energy $T$.

beam is independent of the beam's initial spread and, as Table I shows, decreases with increasing gradient of the stopping power. In the table, the gradient is calculated from the parametrization of the stopping power of hydrogen for $\mu^{-}$from [14], using the measured parameters given in the paper.

Such effects are shared by ionization cooling [5-7], which operates at higher kinetic energies. However, in contrast to ionization cooling, frictional cooling, which operates at low kinetic energies, is greatly affected by nuclear scattering, which contributes to the spread in kinetic energies of the cooled beam to a larger extent than the straggling of energy losses. This is due to particles scattering away from the electric field direction, possibly even in directions opposed to that of the field; they are then slowed down and reaccelerated to the equilibrium energy in the direction of the field.

\section{LOW-ENERGY STOPPING PROCESSES}

At high kinetic energies, a projectile slows down in a medium through excitation and ionization of the medium atoms. One can neglect the interactions with the nuclei of

TABLE I. Energy spreads of frictionally cooled beams of $\mu^{-}$ in $\mathrm{H}_{2}$ gas from [4] and $\mu^{+}$in He; and gradient of the stopping power of $\mathrm{H}_{2}$ for $\mu^{-}$from the parametrization in [14].

\begin{tabular}{|c|c|c|c|c|c|}
\hline$T_{\text {eq }}$ & {$[\mathrm{keV}]$} & 2.0 & 2.5 & 3.0 & \\
\hline$d S / d T$ & {$\left[\mathrm{~cm}^{2} / \mathrm{mg}\right]$} & 7.55 & 6.46 & 5.697 & \multirow{4}{*}{$\mu^{-}$in $\mathrm{H}_{2}$} \\
\hline Total & {$[\mathrm{keV}]$} & 0.60 & 0.69 & 0.83 & \\
\hline Scattering & {$[\mathrm{keV}]$} & 0.57 & 0.64 & 0.76 & \\
\hline Straggling & {$[\mathrm{keV}]$} & 0.18 & 0.27 & 0.34 J & \\
\hline Charge exchange & {$[\mathrm{keV}]$} & 0.07 & 0.11 & 0.17 & $\mu^{+}$in $\mathrm{He}$ \\
\hline
\end{tabular}

the medium and assume that a positively charged projectile is stripped of all its electrons [16,17]. Thus, the stopping power of the medium for positive particles is the same as for negative ones. This is the energy region of ionization cooling, and the energy loss here is comparatively simpler to model than energy loss at low energies.

At low energies, projectiles slow down by Coulombic interactions with both the nuclei and the electrons of the medium; furthermore, the interactions of positively charged projectiles involve more than excitation and ionization $[18,19]$, leading to a difference in the stopping powers for positive and negative projectiles.

Scattering of the projectile particle off of a nucleus results in a loss of energy and a change of direction. Though, as described above, nuclear scattering has the largest impact on the final energy spread of a cooled $\mu^{-}$ beam, it is not the main mechanism of energy loss. The nuclear stopping power is orders of magnitude smaller than the electronic stopping power over the energy range of interest to frictional cooling [13].

\section{Electronic stopping}

The interactions of the projectile ${ }^{2}$ with the electrons of the stopping medium are the dominant mechanisms of energy loss. In the frictional-cooling energy region, these interactions are the excitation and ionization of the medium atoms $(\mathrm{X})$,

$$
\begin{aligned}
\mu^{(+)}+\mathrm{X} & \rightarrow \mu^{(+)}+\mathrm{X}^{*} \\
\mu^{(+)}+\mathrm{X}^{q} & \rightarrow \mu^{(+)}+\mathrm{X}^{q+n}+n \mathrm{e}^{-}
\end{aligned}
$$

and the capture and loss of an electron by the projectile,

$$
\begin{gathered}
\mu^{(+)}+\mathrm{X}^{q} \rightarrow \mu^{(0)}+\mathrm{X}^{q+1} \\
\mu^{(0)}+\mathrm{X}^{q} \rightarrow \mu^{(+)}+\mathrm{X}^{q}+\mathrm{e}^{-} \\
\mu^{(0)}+\mathrm{X}^{q} \rightarrow \mu^{(+)}+\mathrm{X}^{q+1},
\end{gathered}
$$

where we have introduced the notation $\mu^{(q)}$ to represent charge states of $\mu^{+}$as an ion of muonium (Mu):

$$
\begin{gathered}
\mu^{(+)}=\mathrm{Mu}^{+}=\mu^{+}, \quad \mu^{(0)}=\mathrm{Mu}=\mu^{+} \mathrm{e}^{-}, \\
\text {and } \mu^{(-)}=\mathrm{Mu}^{-}=\mu^{+} \mathrm{e}^{-} \mathrm{e}^{-} .
\end{gathered}
$$

It is obvious that stopping of the neutral charge state plays a role in the stopping of the projectile. We must also consider processes (1a)-(1e) with the replacement of $\mu^{(+)}$by $\mu^{(0)}$, and $\mu^{(0)}$ by $\mu^{(-)}$; and processes involving the negative charge state: (1a) and (1b) with the replacement of $\mu^{(+)}$by $\mu^{(-)}$, and double electron capture and loss

$$
\mu^{(+)}+\mathrm{X}^{q} \rightarrow \mu^{(-)}+\mathrm{X}^{q+2}
$$

\footnotetext{
${ }^{2}$ The equations to follow are equally valid with $\mu^{+}$and $\mathrm{Mu}$ replaced by $\mathrm{p}$ and $\mathrm{H}$.
} 


$$
\mu^{(-)}+\mathrm{X}^{q} \rightarrow \mu^{(+)}+\mathrm{X}^{q-2} .
$$

We write the cross sections for processes (1a)-(1h) for $\mu^{(+)}, \mu^{(0)}$, and $\mu^{(-)}$with the notation $\sigma_{q q^{\prime}}(T)$, denoting the total cross section for the interactions taking a muon of charge state $q$ and energy $T$ to a muon of charge state $q^{\prime}$ (accompanied by an energy loss). To be clear, $q$ and $q^{\prime}$ refer to the charge state of the muonium ion $(+, 0,-)$, not to the charge of the muon itself, which remains positive in these purely electromagnetic interactions.

The total stopping power for the projectile is the combination of the individual stopping powers for the different charge states,

$$
\begin{aligned}
S(T) & =\sum_{q} f^{q}(T) S^{q}(T) \\
& =f^{+}(T) S^{+}(T)+f^{0}(T) S^{0}(T)+f^{-}(T) S^{-}(T),
\end{aligned}
$$

where the $f^{q}$ are the equilibrium charge state fractions [20], which are the solutions to

$$
\frac{d f^{q}}{d x} \propto \sum_{q^{\prime}}\left(f^{q^{\prime}} \sigma_{q^{\prime} q}-f^{q} \sigma_{q q^{\prime}}\right)=0, \quad \forall q,
$$

and

$$
\sum_{q} f^{q}=1
$$

Since the $d f^{q} / d x$ are taken at a fixed $T$, the $f^{q}$ are functions of $T$. For the three-state system $\left\{\mu^{(+)}, \mu^{(0)}, \mu^{(-)}\right\}$, the equilibrium charge states are

$$
f^{q}=A^{q} / \sum_{q^{\prime}} A^{q^{\prime}},
$$

where

$$
A^{q} \equiv \sum_{i \neq j \neq q} \sigma_{i j} \sigma_{j q}+\prod_{i \neq q} \sigma_{i q}
$$

By the nature of its measurement [13], the stopping power shown in Fig. 1 is the total stopping power; that is, the left-hand side of (2).

\section{EFFECTIVE CHARGE}

It is clear from Sec. III that when traveling through matter, $\mu^{+}$spends some time as $\mathrm{Mu}$ and $\mathrm{Mu}^{-}$. This changing of charge state will have a significant impact on the frictional-cooling process, which involves the restoration of energy losses by an electric field according to $q E$.

Figure 2 shows the cross sections for the chargeexchange processes of $\mu^{+}$in helium obtained by velocity scaling those for protons in [21]. The cross sections resulting in $\mu^{(-)}$charge states are orders of magnitude smaller than those taking $\mu^{(-)}$to $\mu^{(+)}$or $\mu^{(0)}$. So $\mu^{+}$traveling in

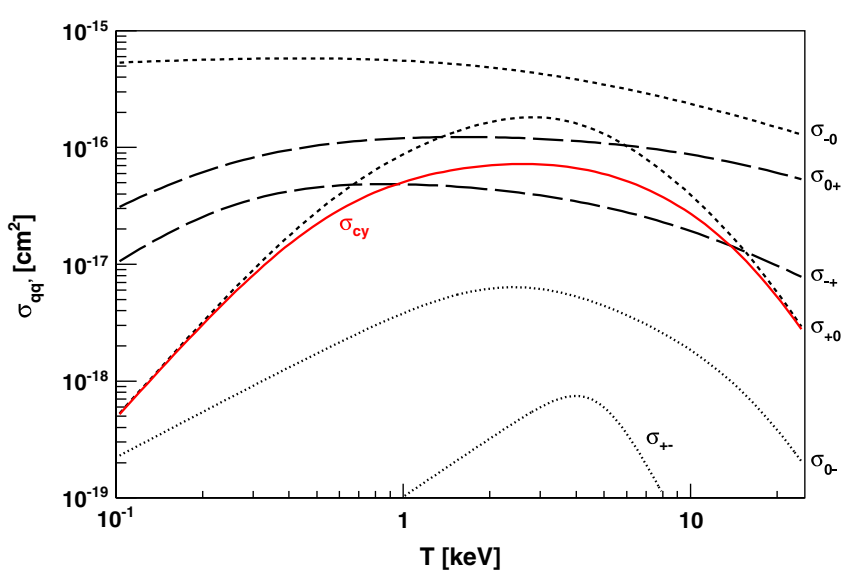

FIG. 2. Charge-exchange cross sections for $\mu^{+}$in helium, velocity scaled from proton cross sections in [21] resulting in final states with $\mu^{(+)}$(large dashes), $\mu^{(0)}$ (small dashes), and $\mu^{(-)}$(dots). The two-state charge-change-cycle cross section is shown in solid red.

helium (and in all the materials we will discuss) is nearly in a two-state system $\left\{\mu^{(+)}, \mu^{(0)}\right\}$. This simplifies the calculation of the equilibrium charge state fractions to

$$
f^{+}=\frac{\sigma_{0+}}{\sigma_{+0}+\sigma_{0+}} \quad \text { and } \quad f^{0}=\frac{\sigma_{+0}}{\sigma_{+0}+\sigma_{0+}} .
$$

The mean-free path for one charge-change cycle $\left(\mu^{(+)} \rightarrow \mu^{(0)} \rightarrow \mu^{(+)}\right)$can be calculated from the meanfree paths for the individual charge-exchange processes, $\lambda_{\text {cy }}=\lambda_{0+}+\lambda_{+0}$. This yields a cross section for a chargeexchange cycle to take place

$$
\sigma_{\mathrm{cy}}=\frac{\sigma_{+0} \sigma_{0+}}{\sigma_{+0}+\sigma_{0+}}
$$

which we show in Fig. 2. Since the charge-exchange interactions take place over distances much shorter than the overall distance the muon travels in the frictional-cooling scheme, we can approximate the charge of the $\mu^{+}$by an effective charge according to

$$
\eta \equiv \sum_{q} q f^{q} .
$$

Figure 3 shows the effective charges of $\mu^{+}$in helium, hydrogen, and neon for both the two-state $\left(\eta_{2}\right)$ and threestate $\left(\eta_{3}\right)$ systems calculated from the charge-exchange cross sections using empirical formulas from [22] fit to measured values [21]. We also show $\eta_{2}$ for carbon; $\eta_{3}$ could not be calculated since the cross sections involving the negative charge state are unknown.

For comparison to the calculated effective charge, we show experimentally measured charge state fractions from [20] for helium, hydrogen, and neon: $f^{+}$, which is the same as $\eta_{2}$; and $\left(f^{+}-f^{-}\right)$, which is the same as $\eta_{3}$. The calculated effective charge matches very well with the measured data. As well, we see that $\eta_{2}$ and $\eta_{3}$ differ 


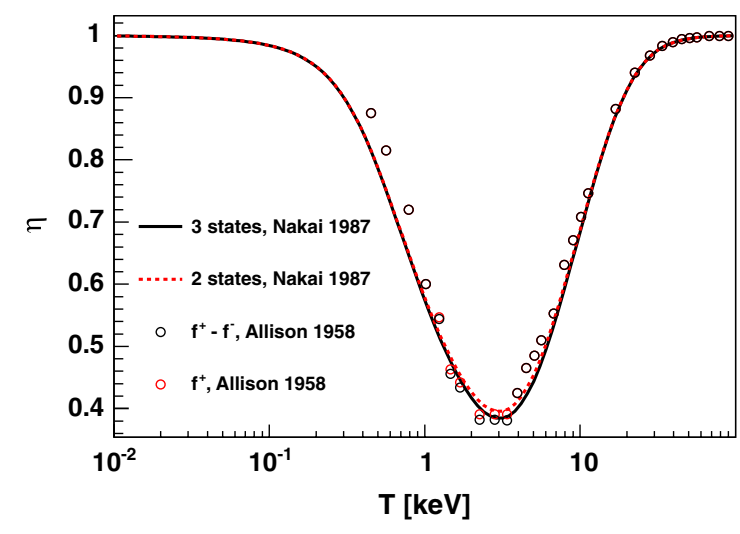

(a) Helium

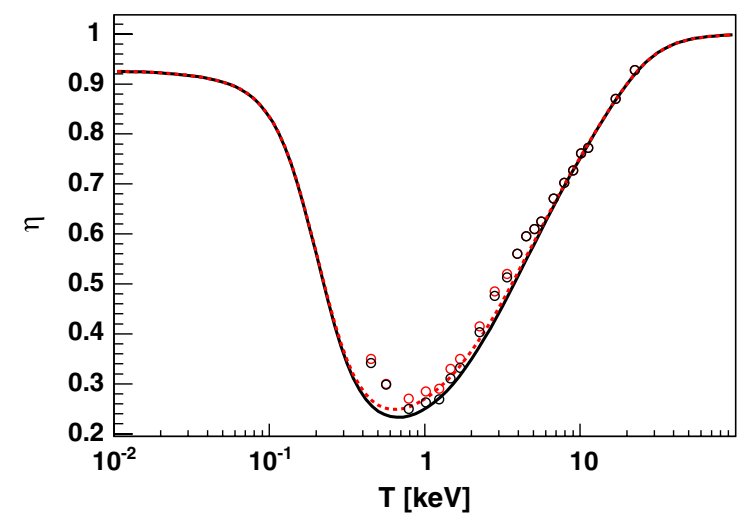

(c) Neon

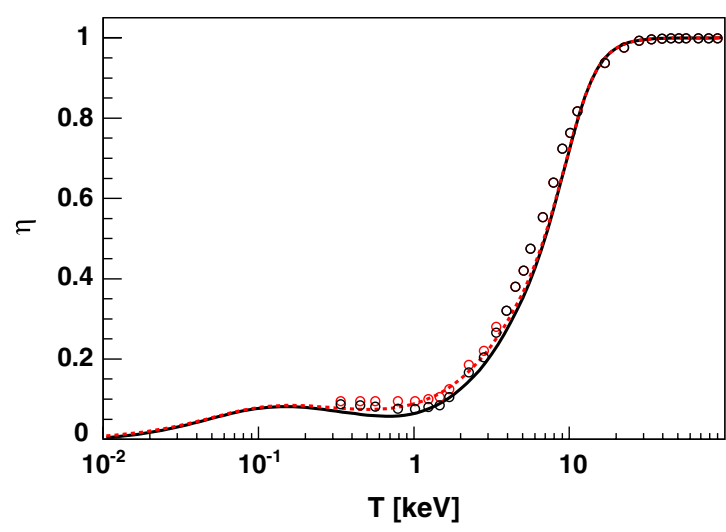

(b) Hydrogen

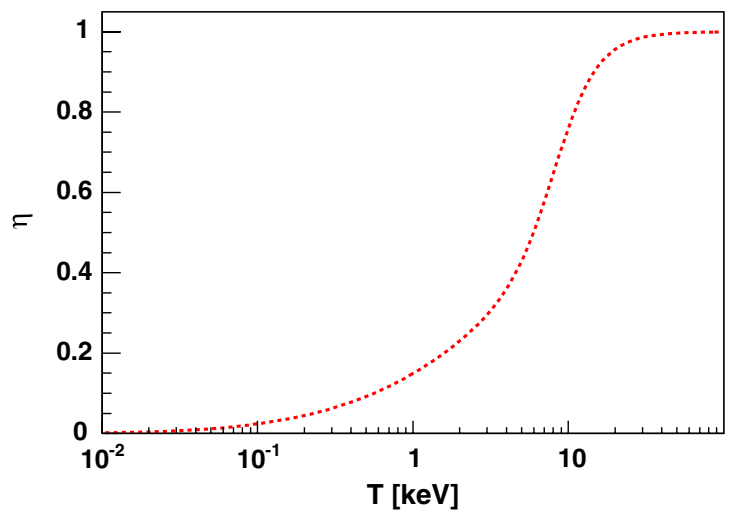

(d) Carbon

FIG. 3. Effective charge (line) for $\mu^{+}$in He, H, Ne, and C for the three-state (solid black) and two-state (dashed red) systems. The data points show the equilibrium charge state fractions taken from [20].

only minutely and only over a small range of energies. The negative charge state fraction contributes to the three-state effective charge at percent level and lower in all three gases.

It is important to note that helium and neon are the only materials in which the effective charge tends to a value of or near unity at low energies. In hydrogen and carbon (as well as water, oxygen, and nitrogen, and therefore air), the effective charge approaches zero at low energies.

\section{ACCELERATING POWER AND COOLING MEDIUM}

The effective charge of a positively charged projectile in the retarding medium of a frictional-cooling scheme can be absorbed into the accelerating power of the electric field. In effect, this makes the accelerating power dependent on the projectile's kinetic energy.

We reformulate here the requirements for frictional cooling stated in Sec. II: An equilibrium energy must be established by balancing energy loss with energy gain,

$$
S\left(T_{\mathrm{eq}}\right)=\frac{E}{\rho} \eta\left(T_{\mathrm{eq}}\right) .
$$

At energies above $T_{\text {eq }}$, the stopping power must be greater than the accelerating power,

$$
S\left(T_{\mathrm{eq}}+\epsilon\right)-\frac{E}{\rho} \eta\left(T_{\mathrm{eq}}+\epsilon\right)>0 .
$$

At energies below $T_{\text {eq }}$, the accelerating power must be greater than the stopping power,

$$
\frac{E}{\rho} \eta\left(T_{\mathrm{eq}}-\epsilon\right)-S\left(T_{\mathrm{eq}}-\epsilon\right)>0 .
$$

The last two requirements can be combined to one statement about the slope of the stopping power relative to that of the accelerating power:

$$
\left.\frac{d S}{d T}\right|_{T_{\mathrm{eq}}}-\left.\frac{E}{\rho} \frac{d \eta}{d T}\right|_{T_{\mathrm{eq}}} \equiv S^{\prime}\left(T_{\mathrm{eq}}\right)-\frac{E}{\rho} \eta^{\prime}\left(T_{\mathrm{eq}}\right)>0 .
$$

All three requirements can be met when

$$
S^{\prime}-S \frac{\eta^{\prime}}{\eta}>0
$$

Accounting for the muon's effective charge greatly reduces the maximum energy at which the condition of (11) 
TABLE II. Maximum energy (in keV) at which a stable equilibrium can be established in a frictional cooling scheme for $\mu^{+}$ with and without accounting for effective charge for several stopping media. A blank entry means no equilibrium energy can be established.

\begin{tabular}{lcccccccccc}
\hline \hline & $\mathrm{He}$ & $\mathrm{H}_{2}$ & $\mathrm{Ne}$ & $\mathrm{C}$ & $\mathrm{N}_{2}$ & $\mathrm{Ar}$ & $\mathrm{H}_{2} \mathrm{O}$ & $\mathrm{O}_{2}$ & $\mathrm{Kr}$ & $\mathrm{Xe}$ \\
\hline Without $\eta$ & 8.4 & 6.2 & 14.1 & 9.6 & 9.0 & 7.9 & 9.0 & 10.7 & 9.0 & 9.0 \\
With $\eta$ & 3.9 & 0.9 & 1.1 & & 0.33 & 0.23 & 4.5 & 3.1 & & \\
\hline \hline
\end{tabular}

is met. Table II lists the cooling ranges for several materials with and without accounting for effective charge.

The effects of charge-exchange processes also reduce the value of $T_{\text {eq }}^{\prime}$, the maximum kinetic energy that can be decelerated to $T_{\text {eq }}$. This effect is illustrated in Fig. 4, which shows the frictional-cooling energy region of the stopping power of helium for $\mu^{+}$. Superimposed on the stoppingpower curve are three electric-field accelerating powers: The black dashed line is the naive accelerating power of Fig. 1, with effective charge neglected, for a field strength $560 \mathrm{kV} \mathrm{cm}^{2} / \mathrm{mg}$ resulting in $T_{\text {eq }}=1 \mathrm{keV}$. The lower red curve is the accelerating power for the same electric field strength, accounting for effective charge. The equilibrium energy is cut in half, but $T_{\text {eq }}^{\prime}$ remains the same. The upper red curve shows the accelerating power accounting for effective charge that results in $T_{\mathrm{eq}}=1 \mathrm{keV}$, which requires a field strength of $970 \mathrm{kV} \mathrm{cm} / 2 \mathrm{mg}$, and reduces $T_{\text {eq }}^{\prime}$ by a factor of 2 .

Achieving the desired equilibrium energy, accounting for effective charge, requires a greater electric field strength than is expected in the naive scheme. The field

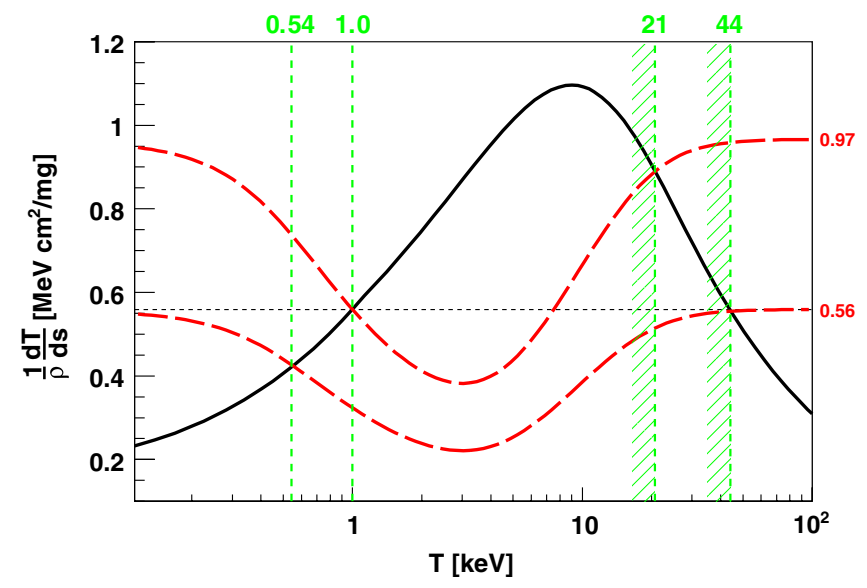

FIG. 4. Frictional-cooling energy region of the stopping power of helium for $\mu^{+}$(solid curve). The horizontal dashed line shows the naive accelerating power neglecting charge-exchange processes of an electric field at $0.56 \mathrm{MV} \mathrm{cm}^{2} / \mathrm{mg}$, resulting in $T_{\text {eq }}=1 \mathrm{keV}$. The two dashed curves show accelerating powers with charge-exchange processes accounted for: the lower curve is for $0.56 \mathrm{MV} \mathrm{cm}^{2} / \mathrm{mg}$, resulting in $T_{\text {eq }}=0.54 \mathrm{keV}$; the upper curve is for $0.97 \mathrm{MV} \mathrm{cm}^{2} / \mathrm{mg}$, resulting again in $T_{\text {eq }}=1 \mathrm{keV}$. strength may in fact have to be an order of magnitude larger, depending on what the desired $T_{\mathrm{eq}}$ and the stopping medium are (Fig. 5). On the bright side, the relative slope of the stopping power is generally larger when effective charge is accounted for, perhaps causing (according to [4]) the final energy spread of a cooled beam to be smaller. As well, the stronger electric fields may reduce the spread caused by scattering.

We performed Monte Carlo simulations of the cooling of $\mu^{+}$beams with discrete charge-exchange interactions, as well as simulations using the effective-charge approximation. These conformed to the calculations presented in Fig. 5 to within a few percent.

From the simulations we can calculate the spread of the beam energy around $T_{\text {eq }}$ due solely to charge-exchange interactions. Table I lists these spreads for $\mu^{+}$in helium at the same equilibrium energies cooled to in [4], which looked at $\mu^{-}$in hydrogen. The spread due to charge exchange for $\mu^{+}$is smaller than the spreads due to scattering and straggling for $\mu^{-}$. A comparison using $\mu^{+}$in hydrogen is not possible because the maximum $T_{\text {eq }}$ in $\mathrm{H}_{2}$ for $\mu^{+}$is $0.9 \mathrm{keV}$.

The choice of stopping medium is even further limited than the requirement that the relative slope of the stopping power be positive. This is illustrated by the example of oxygen, in which the relative slope of the stopping power is just barely positive over a small region of energies; but it is not sufficiently large to clearly establish an equilibrium energy.

Furthermore, at low energies, oxygen's stopping power is proportional to particle velocity, $S \propto T^{1 / 2}$, but its $\eta$ is proportional to $T^{k}$, with $k>\frac{1}{2}$, causing the accelerating power to decrease below the stopping power at low energies. A particle that experiences a large loss of energy in one interaction-or one that scatters into a direction opposite that of the electric field-will not reaccelerate to $T_{\text {eq }}$,

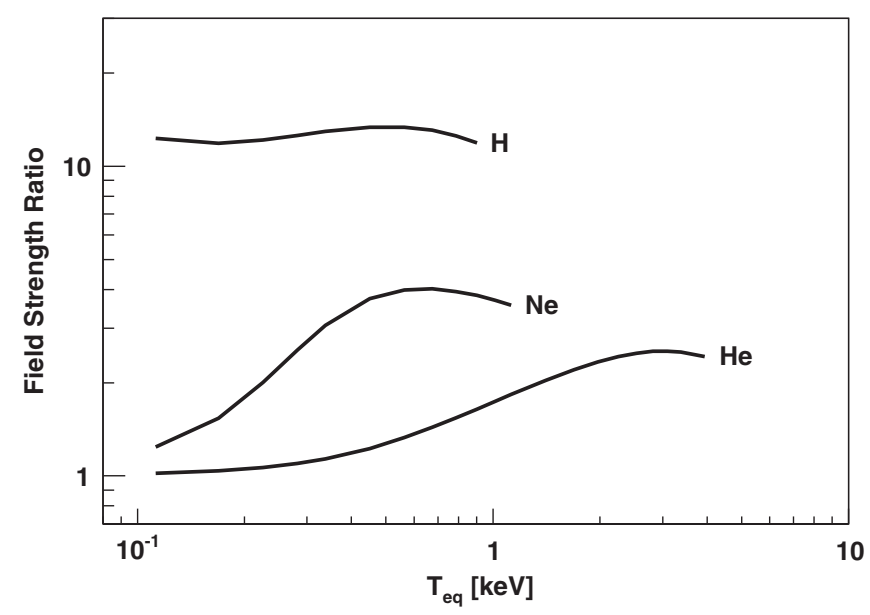

FIG. 5. Ratio of electric field strength required to achieve the desired equilibrium energy accounting for effective charge to the field strength required without accounting for effective charge. 
but rather continue decelerating to thermal energies and be lost to the cooling process. This would severely limit the use of such a medium in a frictional-cooling scheme.

The only viable media for a gaseous frictional-cooling scheme for $\mu^{+}$with equilibrium energies at or above $1 \mathrm{keV}$ are helium and water vapor. For $T_{\text {eq }}$ just below $1 \mathrm{keV}$, hydrogen and neon are also viable $;^{3}$ and for $T_{\text {eq }}$ of a few hundred electron volts, argon and nitrogen are also viable.

\section{BEAM NEUTRALIZATION AND FOIL-BASED SCHEMES}

The frictional-cooling experiment of [11] iterated energy loss and replacement by means of a series of moderating foils with electric fields between them [Fig. 6(a)]. The experiment and a Monte Carlo simulation in [4] gave promising results for such a scheme for the cooling of negative muons. In both publications it was posited that the frictional-cooling scheme used with $\mu^{-}$could also work with $\mu^{+}$; more recently this idea has been revisited in $[23,24]$. However, the presence of charge-exchange interactions greatly limits the yield for such a scheme; in fact, leading to a zero yield.

A foil-based frictional-cooling scheme has the benefit of separating energy loss, occurring in the foils, from energy restoration, occurring between foils. Since particles are reaccelerated in vacuum, the energy restoration depends on the charge state of the particle as it exits the foil. Those exiting in a neutral state are blind to the reacceleration field.

The four studies cited above used and simulated carbon foils in their frictional-cooling schemes, setting the potential difference between foils $(\Delta V)$ to precisely compensate the energy lost in a foil $(\Delta T)$ by a particle with kinetic energy $T_{\text {eq }}$; and assumed every particle exits every foil in a charged state. However, for positively charged particles, after passing through a foil at mean energy $T_{\text {eq }}$, a beam is divided into two populations: a fraction $f^{+}\left(T_{\mathrm{eq}}-\Delta T\right)$ in the $\mu^{+}$state and a fraction $\left(1-f^{+}\right)$in the Mu state [Fig. 6(b)]. Between the foils, a $\mu^{+}$reaccelerates to $T_{\text {eq }}$. Upon exiting the next foil in the cooling series, the $\mu^{+}$ population fraction decreases further to $\left(f^{+}\right)^{2}$. Between the foils, a $\mathrm{Mu}$ atom does not reaccelerate and exits the next foil at an energy even further below $T_{\text {eq }}$, where the probability of losing its electron is even smaller. Furthermore, a Mu atom that loses its electron must exit several foils in a row in the positive state in order to reaccelerate to $T_{\text {eq }}$. This is unlikely to occur. As the beam passes through the array of foils, it is neutralized and slowed down. Cooling becomes an impractical goal.

\footnotetext{
${ }^{3}$ Though Fig. 5 shows the range for $T_{\text {eq }}$ for neon extending up to $1.1 \mathrm{keV}$, in practice, $T_{\text {eq }}$ would have to sit below this upper limit by an amount large enough to accommodate the spread of the beam energy described in Sec. II.
}

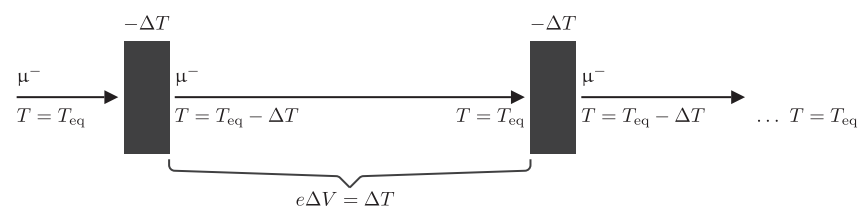

(a)

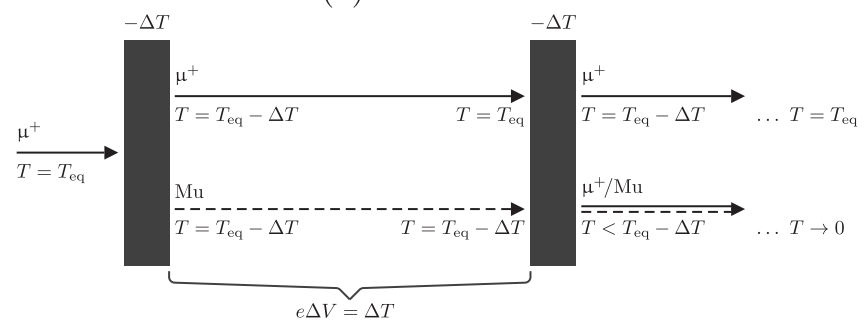

(b)

FIG. 6. Diagram of a foil-based cooling scheme for $\mu^{-}$(a) and $\mu^{+}$(b); the potential difference $(\Delta V)$ between two foils (shown by thick vertical lines) is tuned to restore the energy lost $(\Delta T)$ by a muon with kinetic energy $T=T_{\text {eq }}$.

We performed a Monte Carlo simulation of exactly this mechanism. The foil density was chosen to match the simulations in [4] and the experiment in [11], $5 \mu \mathrm{g} / \mathrm{cm}^{2}$ (25-nm-thick foils of $2-\mathrm{g} / \mathrm{cm}^{3}$ amorphous carbon). Scattering and energy-loss straggling were neglected, since they would only worsen the beam neutralization described above. Figure 7 shows the yield ${ }^{4}$ of the foil-based scheme as a function of the number of foils traversed for monoenergetic beams starting at $9 \mathrm{keV}$, the maximum $T_{\text {eq }}$ possible, and $4 \mathrm{keV}$, the $T_{\text {eq }}$ used in [4]. After a handful of foils, the yield falls off exponentially with the number of foils traversed. After passing through the first foil and energy restoration, the mean energy of the beam is greatly reduced from $T_{\text {eq }}$ and the energy spread is greatly enlarged. The mean energy continues to fall with each successive foil.

We also simulated a scheme in which the foils are coated with frozen noble gas to increase the fraction of particles exiting them in the positive charge state. This is inspired by the scheme for the production of low-energy muons from a surface muon beam at the Paul Scherrer Institute (PSI), which uses frozen argon and krypton [25]. Neither argon nor krypton coatings improve the beam neutralization. A neon coating marginally slows beam neutralization (shown in Fig. 7), but does not prevent the degradation of the beam energy. Moreover, we simulated the charge-exchange effects of the coatings but neglected their impact on energy loss. This assumes that the coatings are nanometers thin; however, the coatings used at PSI are a much thicker

\footnotetext{
${ }^{4}$ In the simulation, tracking of a particle is terminated when it does not have sufficient kinetic energy to pass completely through a single foil according to [13]. The yield is thus defined as the fraction of muons surviving passage through the foil.
} 


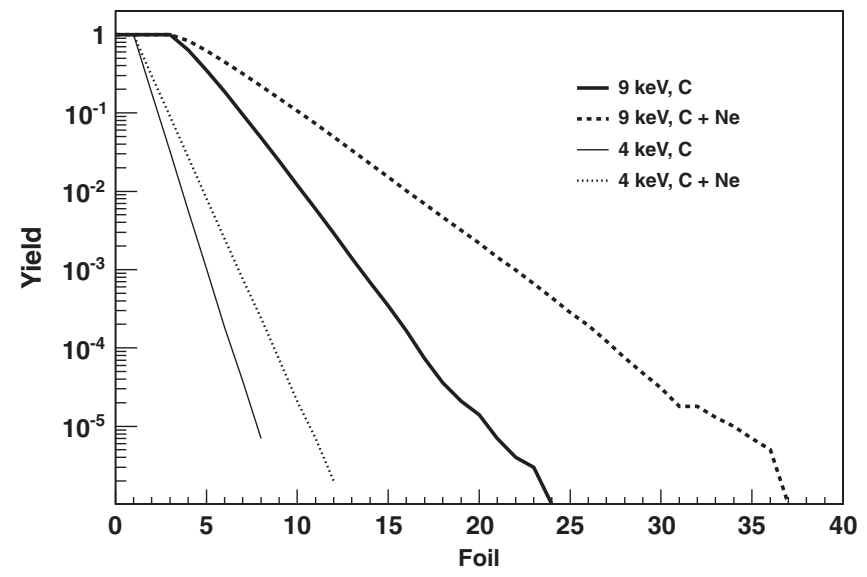

FIG. 7. Yield of $\mu^{+}$in carbon-foil frictional-cooling schemes as a function of number of foils traversed after reaching $T_{\text {eq }}$, for both naked foils and frozen-neon-coated foils.

200-300 nm. Such thicker coatings would hasten beam loss.

The scheme of [23], also based on carbon (graphite) foils, requires the beam to pass through an order of 100 foils at the equilibrium energy. A beam of positively charged muons (or protons) would be unable to survive such a scheme.

\section{CONCLUSION}

Muon collider schemes employing frictional cooling are a viable option for collision of multi-TeV lepton beams. Several articles have been published with analytical and experimental results for frictional cooling of negatively charged particles. Many of these articles have conjectured that the results for positively charged particles will be the same as for negatively charged ones, and schemes for the cooling of positive particles have been proposed. A key group of physics processes involved in the slowing down of positive particles-those changing the charge state-has been neglected in these studies. We found that accounting for these processes significantly alters the results for positive particles from those for negative ones: The choice of cooling medium is greatly limited, such that helium gas becomes the only viable medium; with foil-based schemes completely ruled out. The range of equilibrium energies for the cooled beam is also greatly limited, with a maximum possible energy of approximately $4 \mathrm{keV}$ for $\mu^{+}(36 \mathrm{keV}$ for protons). The electric field strength required to bring a beam of positive particles to an equilibrium energy is significantly greater than the strength required to bring a beam of negative ones to the same energy.

[1] M. M. Alsharo et al., Phys. Rev. ST Accel. Beams 6, 081001 (2003).

[2] C. Ankenbrandt et al., Fermilab Technical Report No. TM-2399-APC, 2009.

[3] H. Abramowicz, A. Caldwell, R. Galea, and S. Schlenstedt, Nucl. Instrum. Methods Phys. Res., Sect. A 546, 356 (2005).

[4] M. Mühlbauer, H. Daniel, and F. J. Hartmann, Hyperfine Interact. 82, 459 (1993).

[5] D. Neuffer, Part. Accel. 14, 75 (1983) [http://inspirehep .net/record/188841].

[6] V. V. Parkhomchuk and A. N. Skrinsky, AIP Conf. Proc. 352, 7 (1996).

[7] C.-x. Wang and K.-J. Kim, Phys. Rev. Lett. 88, 184801 (2002)

[8] R. Palmer et al., Phys. Rev. ST Accel. Beams 8, 061003 (2005).

[9] C. H. Albright et al., arXiv:physics/0411123.

[10] D. Greenwald, in Proceedings of the 2011 Particle Accelerator Conference, NY, USA (IEEE, New York, 2011), pp. 142-144.

[11] M. Mühlbauer et al., Nucl. Phys. Proc. Suppl. 51A, 135 (1996) [http://inspirehep.net/record/434120].

[12] B. H. Bransden and C. Forster, J. Phys. B 23, 115 (1990).

[13] Stopping powers and ranges for protons and alpha particles, 1993, Bethesda, Maryland: ICRU (1993), p. 286 (ICRU report: 49).

[14] M. Agnello et al., Phys. Rev. Lett. 74, 371 (1995).

[15] P. Sigmund and A. Schinner, Eur. Phys. J. C 15, 165 (2001).

[16] U. Fano, Annu. Rev. Nucl. Part. Sci. 13, 1 (1963).

[17] J.F. Ziegler, J.P. Biersack, and M.D. Ziegler, The Stopping and Range of Ions in Matter (SRIM Co., Chester, Maryland, 2008).

[18] M. Senba, J. Phys. B 21, 3093 (1988).

[19] M. Senba, J. Phys. B 22, 2027 (1989).

[20] S. K. Allison, Rev. Mod. Phys. 30, 1137 (1958).

[21] Y. Nakai, T. Shirai, T. Tabata, and R. Ito, At. Data Nucl. Data Tables 37, 69 (1987).

[22] A. E. S. Green and R. J. McNeal, J. Geophys. Res. 76, 133 (1971).

[23] T. J. Roberts and D. M. Kaplan, in Proceedings of the 23rd Particle Accelerator Conference, Vancouver, Canada, 2009 (IEEE, Piscataway, NJ, 2009).

[24] D.M. Kaplan, in Proceedings of DPF-2009 [arXiv:0910.3154].

[25] E. Morenzoni, F. Kottmann, D. Maden, B. Matthias, M. Meyberg, T. Prokscha, T. Wutzke, and U. Zimmermann, Phys. Rev. Lett. 72, 2793 (1994). 